\title{
Emotionales Erleben von Lehrkräften beim Unterrichten
}

\author{
Anne C. Frenzel ${ }^{1}$ und Thomas Götz ${ }^{2}$ \\ ${ }^{1}$ Ludwig-Maximilians-Universität München, ${ }^{2}$ Universität Konstanz \& Pädagogische Hochschule Thurgau
}

\begin{abstract}
Zusammenfassung. Ziel dieser Studie war es, das Ausmaß an Variabilität des Erlebens von Freude, Angst und Ärger bei Lehrkräften zu ermitteln, das auf ihre Persönlichkeit, das Unterrichtsfach (hier: Mathematik vs. Physik) und die jeweils unterrichtete Klasse zurückzuführen ist. Zudem wurde der Einfluss von Person- und Kontextmerkmalen auf das emotionale Erleben beim Unterrichten untersucht. 59 Gymnasiallehrkräfte (9 weiblich) gaben anhand von einem Fragebogen sowie einem Tagebuch Auskunft über ihre Unterrichtsemotionen in jeweils vier ihrer Klassen (zwei Mathematik-, zwei Physikklassen). Mehrebenenanalytische Varianzanalysen zeigten, dass insbesondere Angst, aber auch Freude, stärker person- und weniger kontextspezifisch sind, während Ärger in gleichen Teilen von der Lehrerpersönlichkeit wie von der jeweils unterrichteten Klasse abhängt. Eine Fachspezifität unterrichtsbezogener Emotionen ließ sich bei Kontrolle der Klassenspezifität nicht nachweisen. Mehrebenen-Regressionen lieferten Hinweise darauf, dass das emotionale Erleben der Lehrkräfte von ihren Kontrollüberzeugungen, aber auch von der Größe der Klassen und vom in den jeweiligen Stunden vorherrschenden Verständnis-, Motivations- und Disziplinniveau abhängt. Die Bedeutsamkeit der Befunde für die Unterrichtspraxis wird diskutiert.
\end{abstract}

Schlüsselwörter: Freude, Angst, Ärger, Lehrkräfte, Unterricht

Teachers' Emotional Experiences During Teaching

\begin{abstract}
This study aimed at determining the degree of variability of teachers' emotions during teaching which is attributable to teacher personality, to the subject taught (here: math vs. science), and to the group of students taught. In addition, we analyzed how personal and contextual characteristics influenced the teachers' emotional experiences. 59 secondary school teachers ( 9 female) rated their experience of enjoyment, anxiety, and anger during teaching with a questionnaire and a diary in reference to four different groups of students (two math and science each). Multilevel analysis of variance showed that anxiety and enjoyment were more person- than context-specific, while anger was equally dependent on the teachers' personality and on the specific group of students taught. The emotional experiences during teaching were not subject-specific when controlling for context specificity. Multilevel regression analyses provided evidence that teachers' emotional experiences are related to their locus of control regarding teaching success, but also to class size and to the levels of student understanding, motivation and discipline during the lessons. The relevance of the findings for educational practice are discussed.
\end{abstract}

Keywords: enjoyment, anxiety, anger, teachers, instruction

\section{Einleitung}

Emotionen sind in schulischen Instruktions- und Interaktionsprozessen allgegenwärtig: Lernende ängstigen sich vor Prüfungen oder sind stolz auf ihre Leistungen; Lehrkräfte freuen sich über Lernfortschritte ihrer Schülerinnen und Schüler oder ärgern sich über deren Unaufmerksamkeit, etc. Es ist anzunehmen, dass die Gefühle von Schülerinnen und Schülern, aber auch die von Lehrkräften, einen bedeutsamen Einfluss auf die Qualität von Lehren und Lernen im Schulkontext nehmen (Frenzel, Götz \& Pekrun, im Druck; Meyer \& Turner, 2002; Sutton \& Wheatley, 2003) - auch wenn die Befundlage zum Einfluss emotionalen Erlebens von Lehrkräften auf die Qualität von Lehr-Lernprozessen im Unterricht als noch defizitär zu bezeichnen ist. Lehrkräfte geben in
Interviews ihrer Überzeugung Ausdruck, dass ihre Emotionen die Effektivität ihres Unterrichts beeinflussen (Sutton, 2004). Auch konnten Kunter, Tsai, Brunner und Krauss (2005) zeigen, dass sich Schülerinnen und Schüler von Mathematik-Lehrkräften, die Begeisterung und Freude am Unterrichten erleben, kognitiv und motivational stärker gefördert fühlen und den Unterricht als störungsfreier beschreiben. In einer Studie von Turner, Meyer, Midgley und Patrick (2001) zeigten Schülerinnen und Schüler in Klassenzimmern mit positivem emotionalem Klima (geprägt durch Humor und Freude der Lehrkräfte beim Unterrichten) größere Lernziel-Orientierung und suchten bei Problemen aktiv nach Hilfe, während in Klassen, die durch Zynismus und negative Emotionen der Lehrkräfte geprägt waren, Performanz-Orientierung und Self-Handicapping vorherrschten. 
Jenseits ihrer Wirkungen auf Lehr-Lernprozesse können Emotionen auch als wichtige Outcomevariablen per se beispielsweise hinsichtlich subjektiven Wohlbefindens angesehen werden (z. B. Hascher, 2004). Emotionales Belastungserleben ist gerade im Lehrerberuf eine bedeutungsvolle Problematik (z. B. Schaarschmidt, 2004). Trotzdem fristen Schüler- und Lehreremotionen neben prominenteren Themen wie zum Beispiel Leistungsmotivation auf Schülerseite oder auch generellem Stress und Belastungserleben auf Lehrerseite bisher eher ein Schattendasein in der Lehr-Lernforschung. Verschiedene Faktoren mögen hierfür verantwortlich sein. Zum einen wurden Emotionen konzeptuell in manchen Theorietraditionen eher als Epiphänomene oder bestenfalls als Resultate von Motivations- oder Kognitionsprozessen angesehen, nicht aber als eigenständige Konstrukte. Zum anderen sind Emotionen generell aufgrund ihrer flüchtigen und fast ausschließlich auf subjektivem Erleben basierenden Natur empirisch nur schwer zugänglich, was eine wissenschaftliche Auseinandersetzung mit ihnen erschwert. Um uns diesen komplexen Konstrukten zu nähern, wählten wir in der vorliegenden Studie einen multimethodalen $\mathrm{Zu}$ gang: Wir erfassten Lehreremotionen zum einen in Form von Tagebüchern, in denen wir die Lehrkräfte baten, ihr emotionales Erleben im Klassenzimmer direkt im Anschluss an konkrete Unterrichtsstunden zu beurteilen; und zum anderen in Form von Fragebogen, in denen Lehrkräfte $\mathrm{zu}$ ihrem Erleben beim Unterrichten im Allgemeinen Auskunft geben sollten. Damit wurden wir einer Konzeptualisierung von Emotionen auf zweierlei Weise gerecht: Einerseits als «States», d. h. flüchtigen, situationsspezifischen Reaktionen auf spezifische Umweltbedingungen, und andererseits als «Traits», den persönlichkeitsbedingten Neigungen, auf Situationen wiederholt mit spezifischen emotionalen States zu reagieren (z. B. Lazarus, 1994). Auch wenn zu erwarten ist, dass allein aufgrund der Unterschiedlichkeit der Methoden die Ergebnisse voneinander abweichen (Methodenvarianz), gehen wir davon aus, mit beiden Ansätzen Gleiches zu messen: Die Summe der über einen längeren Zeitraum hinweg gemittelten emotionalen States sollte ebenfalls Trait-Emotionen reflektieren (vgl. z. B. Hedges, Jandorf \& Stone, 1985; Parkinson, Briner, Reynolds \& Totterdell, 1995; Thomas \& Diener, 1990).

Auf Schülerseite gibt es insbesondere durch die Arbeiten von Pekrun und Kollegen (z. B. Pekrun, Götz, Titz \& Perry, 2002) inzwischen kumulative empirische Evidenz bezüglich Phänomenologie, Struktur, Ursachen und Wirkungen von diskreten Emotionen in Lern- und Leistungssituationen (u. a. Freude, Stolz, Ärger, Langeweile, Hoffnungslosigkeit). Zudem liegen inzwischen reliable und valide Fragebogen zur Erfassung dieser Emotionen vor (z. B. «Academic Emotions Questionnaire» [AEQ], Pekrun, Götz \& Perry, 2005; sowie «Academic Emotions Questionnaire - Mathematics» [AEQM], Pekrun, Götz \& Frenzel, 2005; siehe auch Ramm et al., 2006, zum Einsatz der deutschsprachigen Skalen zu Schüler- emotionen des AEQ-M im Rahmen der nationalen Ergänzungsstudien von PISA 2003).

Auf Lehrerseite dagegen gibt es zwar recht gut etablierte Skalen für die Erfassung von Belastungserleben und Burnout (Maslach \& Jackson, 1986; Maslach, Schaufeli \& Leiter, 2001), den Autoren sind aber kaum Instrumente bekannt, die auf die Erfassung qualitativ unterschiedlicher Emotionen wie Freude, Ärger oder Angst beim Unterrichten zielen. Eine Ausnahme bildet das «Survey of Feelings about Teaching» zur Erfassung von Angst und Sorgen beim Unterrichten (Payne \& Manning, 1990), das jedoch in nur einer einzigen publizierten Studie zum Einsatz kam. Die Mehrzahl der bestehenden Untersuchungen zu Emotionen von Lehrkräften war bisher vorwiegend qualitativ orientiert; quantitative Daten sind kaum vorhanden. Die bestehenden Studien zeigen, dass Freude und Ärger zu den am häufigsten und intensivsten von Lehrkräften erlebten bzw. berichteten Emotionen zählen (vgl. Sutton \& Wheatley's Review zu «Teacher Emotions», 2003), aber auch Angst beim Unterrichten insbesondere bei jungen bzw. sich noch in Ausbildung befindenden Lehrkräften in nicht unerheblichem Maße auftritt (z. B. Grimminger, 2005; Payne \& Manning, 1990). Implizit wird in vielen dieser Studien von der Existenz emotionaler Traits von Lehrkräften ausgegangen, die das emotionale Klima im Unterricht prägen. Bisher fehlt jedoch ein empirischer Nachweis dazu, inwieweit das Erleben von Emotionen beim Unterrichten ausschließlich auf Unterschiede zwischen Personen zurückgeht, oder aber durch situative Bedingungen beeinflusst wird (schwierige vs. angenehme Klassen, komplexer vs. «dankbarer» Stoff, etc.).

Im Zentrum dieser Studie stand daher die Frage nach Antezedenzien von Lehreremotionen. Zunächst explorierten wir, zu welchem Ausmaß die Variabilität des emotionalen Erlebens beim Unterrichten von der Persönlichkeit der Lehrkraft, vom Unterrichtsfach oder von der jeweils unterrichteten Klasse abhängt. Basierend auf der Annahme, dass emotionales Erleben in Abhängigkeit von Person und Situation fluktuiert, strebten wir außerdem an, zu untersuchen, inwieweit Person- und Kontextmerkmale (z. B. Lehr-Erfahrung, Größe der Klassen, etc.) diese inter- und intraindividuelle Variabilität erklären können. Eine Analyse möglicher Ursachen von nicht nur interindividueller, sondern auch intraindividueller Variabilität ist insbesondere bei intraindividuell fluktuierenden Merkmalen wie Emotionen von großem Interesse und notwendig, um Fehlschlüsse zu vermeiden (wenn beispielsweise Variablen bei interindividueller Analyse positiv korrelieren, innerhalb von Personen aber negativ; vgl. Schmitz, 1987). Erkenntnisse zur Spezifität emotionalen Erlebens implizieren zudem, wie sinnvoll Interventionen verschiedener Generalisierungsgrade sein können - so argumentieren beispielsweise Götz, Frenzel, Pekrun, Hall und Lüdtke (in press), dass ihre Befunde zur Fachspezifität emotionalen Erlebens von Schülern dafür sprechen, anstatt einer generellen Therapie von Prüfungsangst eher gezielt gegen «MathematikAngst» oder «Sprachen-Angst» vorzugehen. 


\subsection{Unterrichtsbezogene Lehreremotionen: Person-, Fach- und Klassenspezifität}

Da zum emotionalen Erleben beim Unterrichten kaum empirische Evidenz vorliegt, leiteten wir unsere diesbezüglichen Überlegungen und Hypothesen aus verschiedenen verwandten Forschungsbereichen ab. Hinsichtlich der Konzeptualisierung von Emotionen als Persönlichkeitseigenschaften (Traits) oder als situationsspezifische «States» sind (1) Erkenntnisse aus der allgemeinen Emotionsforschung relevant. Annahmen zu einer möglichen Fachspezifität emotionalen Erlebens beim Unterrichten leiteten wir aus (2) Arbeiten zu Lern- und Leistungsemotionen sowie (3) Studien zu Lehrer-Selbstwirksamkeit ab. Letztere liefern darüber hinaus Hinweise dazu, dass Emotionen von Lehrkräften klassenspezifisch ausgeprägt sein könnten.

(1) In der allgemeinen Emotionsforschung wird seit langem eine Konzeptualisierung von Emotionen einerseits als «States» und andererseits als «Traits» postuliert (z. B. Trait-Angst, Trait-Ärger). Die latente State-Trait-Theorie (z. B. Steyer, Schmitt \& Eid, 1999) geht davon aus, dass Erhebungen psychischer Befindlichkeiten stets sowohl von der Person als auch von der Situation beeinflusst werden und dass diese separate Varianzquellen individueller Scores in Fragebogen darstellen. In Anlehnung an eine solche Konzeptualisierung zielten wir in dieser Studie unter anderem darauf ab, die Anteile der Varianz berichteter Emotionen zu ermitteln, die zwischen Personen (aufgrund emotionaler Traits) und innerhalb von Personen (aufgrund systematischer Variation der Situationen) liegen.

(2) Jenseits einer Differenzierung zwischen Trait und State ist die Kontextspezifität emotionalen Erlebens Gegenstand wissenschaftlicher Untersuchungen. Ein für unsere Fragestellung bedeutsamer Befund der neueren Forschung im Bereich Lern- und Leistungsemotionen ist, dass diese weitgehend domänen- bzw. fachspezifisch organisiert sind (z. B. Götz, Frenzel, Pekrun \& Hall, 2006; Götz et al, in press). Appraisaltheoretische Ansätze zu Emotionen legen nahe, dass kognitive Bewertungen der Situation (unter anderem hinsichtlich der Sicherheit ihres Eintretens, der Valenz der Situation, aber auch der personellen Ressourcen zur Bewältigung der Situation) bedeutsam für Qualität und Intensität emotionalen Erlebens sind (z. B. Lazarus \& Folkman, 1984; Pekrun et al., 2007; Roseman, Antoniou \& Jose, 1996). Demgemäß ist eine Fachspezifität emotionalen Erlebens von Schülerinnen und Schülern theoretisch erwartbar, da die verschiedenen Unterrichtsfächer von diesen sowohl als unterschiedlich schwierig und wichtig wahrgenommen werden als auch ihr Selbstkonzept von Fach zu Fach variiert (was dementsprechend die kognitiven Einschätzungen beispielsweise der Sicherheit des Eintretens und der Valenz von Erfolg und Misserfolg in den verschiedenen Fächern unterschiedlich ausfallen lässt). So berichtet Marsh (1990) beispielsweise, dass das Selbstkonzept von Schülerinnen und Schülern selbst in eng verwandten Fächern wie Mathematik und Physik nur zu $r=.41$
(Klassen 5/6) bzw. $r=.55$ (Klassen 7-10) korreliert. Hinsichtlich der Fachspezifität emotionalen Erlebens von Schülerinnen und Schülern berichten Götz et al. (in press), dass die Angst in Mathematik und Physik zu $r=.61$ (8. Klasse) bzw. $r=.58$ (11. Klasse) korreliert, die Freude in diesen beiden Fächern zu $r=.40$ (8. Klasse) bzw. $r=.52$ (11. Klasse).

Gleichermaßen ist für Lehrkräfte denkbar, dass sie ihre persönlichen Ressourcen zur Bewältigung konkreter Unterrichtssituationen in den verschiedenen Fächern unterschiedlich wahrnehmen, ihre Selbstwirksamkeit also über Fächer hinweg variiert (vgl. nächsten Absatz). Dementsprechend wäre auch für Lehrkräfte zu erwarten, dass ihr emotionales Erleben beim Unterrichten vom jeweils unterrichteten Fach abhängig ist (vgl. auch Pekrun et al., 2007). Empirische Evidenz zu dieser Annahme liegt unseres Wissens jedoch nicht vor.

(3) Überlegungen zum emotionalen Erleben beim Unterrichten können auch aus der Forschung zu LehrerSelbstwirksamkeit (Teaching Efficacy) abgeleitet werden. Appraisaltheoretisch ist die Selbstwirksamkeit als kognitive Antezedenz von Emotionen anzusehen (z. B. Pekrun, 2006). Ähnlich wie im Bereich selbstbezogener Kognitionen von Schülern wird in manchen Ansätzen zur LehrerSelbstwirksamkeit davon ausgegangen, dass dieses Konstrukt domänenspezifisch organisiert ist. So regten z. B. Riggs und Enochs (1990) eine fachspezifische Konzeptualisierung von Lehrer-Selbstwirksamkeit an und entwickelten das explizit auf das Unterrichten von Naturwissenschaften bezogene «Science Teaching Efficacy Beliefs Instrument»(STEBI), welches häufig bezüglich weiterer Fächer, unter anderem auch für das Fach Mathematik, modifiziert wurde (z. B. Enochs, Smith \& Huinker, 1999). Die Annahme einer Fachspezifität von Unterrichts-Selbstwirksamkeit scheint jedoch bisher eine rein theoretische zu sein. Es ist uns keine Studie bekannt, die empirisch überprüft hat, ob sich Lehrkräfte, die mehrere Fächer unterrichten, in diesen unterschiedlich wirksam bzw. erfolgreich einschätzten, d. h. ob es intraindividuelle Varianz hinsichtlich der Unterrichts-Selbstwirksamkeit gibt, die auf das jeweilig unterrichtete Fach zurückgeht.

Es gibt vereinzelte Studien, die einer möglichen intraindividuellen Varianz von Unterrichts-Selbstwirksamkeit nachgingen (Raudenbush, Rowan \& Cheong, 1992; Ross, Cousins \& Gadalla, 1996). Diese Studien untersuchten jedoch Fachlehrer, sodass bei ihnen die mögliche Quelle intraindividueller Varianz aufgrund von unterschiedlichen Unterrichtsfächern nicht gegeben war. Übereinstimmendes Ergebnis dieser Studien ist, dass die jeweils unterrichtete Klasse eine wichtige Rolle spielt. Mittels Mehrebenenanalysen ermittelten Ross et al. (1996) an einer Stichprobe von 92 Sekundarschul-Lehrerkräften einen Anteil von $21 \%$ der Gesamtvarianz der Selbstwirksamkeitsscores, der auf Varianz innerhalb der Lehrkräfte (zwischen deren verschiedenen Klassen) zurückging. Raudenbush et al. (1992) konnten bei der Mehrebenen-Analyse von 315 Sekundarschul-Lehrerkräften sogar einen Anteil von $44 \%$ der Ge- 
samtvarianz ausmachen, der auf intraindividuelle Variabilität zurückzuführen war.

\subsection{Ursachen inter- und intraindividueller Variabilität emotionalen Erlebens beim Unterrichten}

Basierend auf der Annahme, dass emotionales Erleben von Lehrkräften zum einen person-, aber auch situationsspezifisch ist, stellt sich die Frage, welche Facetten der Lehrerpersönlichkeit und der Situationen das Erleben von Freude, Angst und Ärger beim Unterrichten beeinflussen. Was charakterisiert also Lehrkräfte, die beim Unterrichten verstärkt Angst erleben? Welche Eigenschaften haben Klassen, in denen Lehrkräfte mehr Freude am Unterrichten haben? Aufgrund des Mangels an Forschungsarbeiten zum emotionalen Erleben von Lehrkräften gibt es auch hier kaum konkrete Hinweise in der bestehenden Literatur, vereinzelt lassen sich jedoch Annahmen ableiten.

Hinsichtlich möglicher Prädiktoren auf Personebene ziehen wir Lehr-Erfahrung und Kontrollüberzeugungen als bedeutsam in Betracht. In der Literatur wird Lehrerangst vorwiegend bei jungen Lehrkräften thematisiert (z. B. Hart, 1987; Payne \& Manning, 1990), was impliziert, dass diese Emotion in dieser Altersgruppe verstärkt auftritt. Die Befunde zum Zusammenhang von Lehr-Erfahrung und Lehrer-Selbstwirksamkeit sind uneinheitlich; es wird von Anstiegen (Hoy \& Woolfolk, 1993), Konstanz (Pigge \& Marso, 1993) und sogar Verlusten (Brown \& Gibson, 1982) im Laufe der Dienstjahre berichtet. Dementsprechend würden wir tentativ annehmen, dass auf Personebene das Dienstalter insofern eine Rolle spielt, als Lehrkräfte mit zunehmender Erfahrung weniger Angst erleben, halten es aber für eine offene Frage, inwieweit sich das Dienstalter auf das Erleben von Freude und Ärger beim Unterrichten auswirken mag.

Als weiteren globalen Einflussfaktor auf das emotionale Erleben von Lehrkräften sehen wir ihre Kontrollüberzeugungen an. In Übereinstimmung mit frühen Konzeptualisierungen von Lehrer-Selbstwirksamkeit im Sinne eines «Locus of Control» (z. B. Armor et al., 1976; Ashton \& Webb, 1986) gehen wir davon aus, dass Lehrkräfte sich in den Überzeugungen zu ihren Einflussmöglichkeiten auf das Erreichen von Unterrichtszielen unterscheiden - d. h. wie viel Handlungsspielraum sie sich selbst und Schulen zugestehen und wie viel sie auf externale, unkontrollierbare Umweltfaktoren zurückführen. Appraisaltheoretisch ist die subjektive Einschätzung der Kontrollierbarkeit von Situationen als wichtige Determinante für das Erleben von Emotionen anzusehen (z. B. Pekrun, 2006; Roseman et al., 1996). Das Erleben von Angst und Ärger sollte dementsprechend bei Personen mit externalen Kontrollüberzeugungen stärker ausgeprägt sein, wohingegen ein internaler
«Locus of Control» das Erleben von Freude begünstigen sollte.

Auf Klassenebene halten wir die Faktoren Klassengröße, Anteil an Jungen in der Klasse, Klassenstufe sowie das subjektiv durch die Lehrkraft eingeschätzte Leistungsniveau der Klassen als potenziell bedeutsam für das emotionale Erleben der Lehrkräfte. In der Literatur wird konsistent berichtet, dass Lehrkräfte bevorzugt kleinere Klassen unterrichten und sich in diesen subjektiv effektiver erleben (z. B. Shapson, Wright, Eason \& Fitzgerald, 1980; Smith \& Glass, 1979). Einzig Raudenbush et al. (1992) fanden in ihren Analysen zu intraindividueller Variabilität von Lehrer-Selbstwirksamkeit, dass sich Lehrkräfte in größeren Klassen als selbstwirksamer erlebten als in kleineren. Hinsichtlich möglicher Effekte des Geschlechterverhältnisses in Klassen gibt es zwar keine Untersuchungen, es wird aber konsistent berichtet, dass Jungen im Unterricht häufiger ermahnt und getadelt werden (vgl. z. B. die Meta-Analyse von Jones \& Dindia, 2004) und Mädchen von Lehrkräften als motivierter, wohlerzogener und weniger anspruchsvoll wahrgenommen werden (Davies \& Brember, 1995). Dementsprechend erwarten wir, dass sich der Anteil an Jungen ebenso wie die Größe der Klasse mindernd auf das Erleben von Freude und verstärkend auf das Erleben von Angst und Ärger auswirkt. Schließlich berichten Raudenbush et al. (1992) ausgeprägte positive Effekte des «tracks» (d. h. des Kursniveaus) auf die Selbstwirksamkeit von Lehrkräften. Daraus schließend erwarteten wir, dass das emotionale Erleben in leistungsstarken Klassen positiver ist (mehr Freude, weniger Angst und Ärger).

Schließlich stellt sich die Frage, wie einzelne Unterrichtsstunden charakterisiert sein müssen, damit bei Lehrkräften diskrete Emotionen verstärkt auftreten. Wir gehen davon aus, dass eine Lehrkraft ihren Unterricht dann als erfolgreich erlebt und mit erhöhter Freude und geringerer Angst und geringem Ärger reagiert, wenn (a) die Schüler den Stoff der jeweiligen Stunde verstehen und Lernzuwächse zu verzeichnen sind, (b) die Schüler die Unterrichtsinhalte interessant finden und motiviert am Unterricht teilnehmen, und (c) die Schüler diszipliniert sind und die Klassenregeln befolgen. Diese drei Aspekte halten wir in Anlehnung an in der Unterrichtsforschung genannte Kriterien von Unterrichtsqualität (kognitiver Anspruch, Motivierung sowie Klassenführung, z. B. Helmke, 2003) für bedeutsam. Auch konzeptualisieren Tschannen-Moran und Hoy (2001) Lehrer-Selbstwirksamkeit entlang dieser drei Facetten (selbsteingeschätzte Kompetenz zur Verständnisvermittlung, zur Anregung von Schüler-Engagement und zum «Classroom Management»). Die Annahme stützend, dass diese drei Aspekte mit dem emotionalen Erleben von Lehrkräften im Zusammenhang stehen, nennen Lehrkräfte in qualitativen Studien häufig Schülerlernerfolge sowie Begeisterung und Engagement von Seiten der Schüler als Ursachen von Freude (z. B. Hargreaves, 2000; Zembylas, 2002). Disziplinschwierigkeiten werden dagegen als häufigste Ursache von Ärgererleben genannt (Hargreaves, 2000; Sutton \& Wheatley, 2003). Außerdem konnte Hart 
(1987) zeigen, dass Störungen im Unterricht bei Junglehrern mit dem Erleben von Angst positiv korrelieren.

\subsection{Ziele und Hypothesen der vorliegenden Studie}

Zusammenfassend verfolgten wir mit der vorliegenden Studie zweierlei Ziele. Zum einen strebten wir an, die Anteile inter- und intraindividueller Variabilität von Freude, Angst und Ärger beim Unterrichten zu bestimmen. Zum anderen zielten wir darauf ab, erste Einblicke darin zu gewinnen, inwieweit Person-, Klassen- und Situationsmerkmale diese inter- und intraindividuellen Fluktuationen emotionalen Erlebens erklären können. Basierend auf der Konzeptualisierung von Emotionen als «affektive Traits» nahmen wir an, dass es bei Lehrkräften interindividuell unterschiedlich ausgeprägte Neigungen gibt, beim Unterrichten emotional zu reagieren. Zugleich erwarteten wir aber auch, dass das emotionale Erleben von Lehrkräften über die verschiedenen von ihnen unterrichteten Fächer und innerhalb dieser Fächer auch über die Klassen hinweg variiert. Es war uns ein wichtiges Anliegen, bei der Betrachtung der intraindividuellen Variabilität emotionalen Erlebens beim Unterrichten sowohl das Fach als auch die Klasse als mögliche Quellen von Varianz separat zu berücksichtigen. Im deutschen Sekundarschulsystem ist dies möglich, weil Lehrkräfte in der Regel mindestens zwei Unterrichtsfächer und diese in verschiedenen Klassen unterrichten. Allerdings gibt es hier nur eine eingeschränkte Kombination an Fächern, die typischerweise von einer Lehrkraft unterrichtet werden. Eine häufige Konstellation ist die Verknüpfung von Mathematik und Physik. Obwohl sich diese beiden Fächer inhaltlich nahe sind, generierten wir basierend auf den Befunden zur Domänenspezifität von Emotionen bei Schülern sowie basierend auf der Tatsache, dass «Teaching Science» und «Teaching Mathematics» in der Unterrichts-Selbstwirksamkeitsforschung explizit differenziert betrachtet werden, die Annahme, dass sich Lehrkräfte beim Unterrichten von Mathematik und Physik unterschiedlich fühlen.

Aus vereinzelten qualitativen und quantitativen Studien zu Lehreremotionen sowie zu Lehrer-Selbstwirksamkeit generierten wir außerdem erste Annahmen, welche Personund Situationsmerkmale für diese intra- und interindividuellen Fluktuationen verantwortlich sein könnten. Wir nahmen an, dass auf Personebene Lehr-Erfahrung Angst beim Unterrichten mindern könnte und Kontrollüberzeugung Freude erhöhen sowie Angst und Ärger senken würden. Auf Klassenebene erwarteten wir negative Effekte der Größe der Klassen sowie des Anteils an Jungen in den Klassen auf das emotionale Erleben (weniger Freude, mehr Angst und Ärger), hingegen positive Effekte des Leistungsniveaus der Klassen auf die Emotionen der Lehrkräfte. Schließlich vermuteten wir, dass die Eindrücke der Lehrkräfte hinsichtlich Verständnis, Motivation und Disziplin der Schülerinnen und Schüler in den konkreten Unterrichtsstunden das Erleben von Freude beim Unterrichten erhöhen und die Angst und den Ärger beim Unterrichten senken würden.

\section{Methode}

\subsection{Stichprobe und Design}

Unsere Daten basieren auf einem Convenience-Sample, das $N=59$ Lehrkräfte (50 männlich/9 weiblich) aus 16 Gymnasien des Stadtgebiets und Landkreises München umfasste. Lehrkräfte, die sich noch im Referendariat befanden, wurden bei der Stichprobenrekrutierung ausgeschlossen. Das durchschnittliche Alter lag bei 49.94 Jahren $(S D=$ 11.03; $\operatorname{Min}=27$, Max $=61$ ). Das Dienstalter variierte zwischen 1 und 35 Jahren $(M=16.85 ; S D=11.14)$. Die Geschlechts- und Altersstruktur der Stichprobe reflektiert jene der Population von Mathematik-/Physiklehrern in Bayern (Bayerisches Staatsministerium für Unterricht und Kultus, 2005). Die Teilnahme war für die Lehrkräfte freiwillig (es gab keine Bezahlung oder materielle Entlohnung) und fand unter Einwilligung der jeweiligen Schulleiter statt.

Um mögliche Effekte der Person, des unterrichteten Faches und der Klassen auf das emotionale Erleben getrennt untersuchen zu können, wurde folgendes Design realisiert: Die Befragungen bezogen sich auf vier verschiedene von den Lehrkräften unterrichtete Klassen, wobei jeweils zwei davon Mathematik- und zwei Physik- (bzw. in der Unterstufe Natur und Technik-) Klassen waren. Da Lehrkräfte an Gymnasien typischerweise insgesamt 6 bis maximal 8 Klassen in ihren beiden Unterrichtsfächern unterrichten, implizierte diese genaue Vorgabe geringe Wahlmöglichkeiten hinsichtlich der beteiligten Klassen. Unterrichteten Lehrkräfte mehr als zwei Klassen in einem der beiden Fächer, war ihnen freigestellt, welche sie als Zielklassen für die Befragungen auswählten. Lehrkräfte, deren unterrichtete Klassen diese Konstellation (mindestens zwei Mathematik- und zwei Physikklassen) nicht aufwiesen, waren ebenfalls bei der Stichprobenrekrutierung ausgeschlossen worden. In der realisierten Stichprobe befand sich insgesamt 44 mal die Kombination zwei Klassen Mathematik/zwei Klassen Physik, 12 mal die Kombination zwei Klassen Mathematik/eine Klasse Physik/eine Klasse Natur und Technik (kurz NuT) und drei mal die Kombination zwei Klassen Mathematik/zwei Klassen NuT. Die 236 berücksichtigten Klassen verteilten sich in etwa gleichmäßig auf alle am Gymnasium vorhandenen Klassenstufen (5 bis 13).

\subsection{Befragungen - Ablauf und Inhalt}

Die Befragungen setzten sich aus einer Vorbefragung und einer Tagebuchphase zusammen. Im Rahmen der Vorbefragung wurden die beteiligten Lehrkräfte gebeten, zu Hause 
einen Fragebogen binnen einer Woche zu bearbeiten. Im Anschluss daran folgte die Tagebuchphase, in der die Lehrkräfte über den Zeitraum von zwei Schulwochen jeweils direkt im Anschluss an ihre Unterrichtsstunden in den vier beteiligten Zielklassen ihr emotionales Erleben beschrieben.

Der Fragebogen der Vorbefragung gliederte sich in drei Teile. In Teil 1 wurden die Lehrkräfte zunächst zu ihren Kontrollüberzeugungen befragt. In Anlehnung an die Arbeiten der «Rand-Studien» (Armor et al., 1976) zu Kontrollüberzeugungen von Lehrkräften lautete hier die Instruktion: «Bestimmte Ziele in Ihrem Unterricht zu erreichen, liegt nicht unbedingt nur in Ihrer Hand. Wie viel Einfluss glauben Sie im Allgemeinen auf folgende Dinge zu haben?» Es folgten sechs Items (z. B. «darauf, dass die Schüler den Stoff verstehen»; «darauf, dass sich die Schüler für die Inhalte interessieren»), welche die Lehrkräfte anhand einer fünfstufigen Skala («gar kein Einfluss» bis «sehr viel Einfluss») beantworteten. Die Skala erwies sich als hinreichend intern konsistent (Cronbach's $\alpha=$.82). Im zweiten Teil des Fragebogens berichteten die Lehrkräfte ihr emotionales Erleben getrennt für die Fächer Mathematik und Physik (z. B. «Das Unterrichten von Mathematik [Physik] macht mir Freude»; «Beim Unterrichten von Mathematik [Physik] bin ich in der Regel angespannt und nervös»; «Während meines Mathematik-[Physik-]Unterrichts habe ich oft Grund, mich zu ärgern»). Darauf folgte eine klassenspezifische Erfassung, d. h. die Lehrkräfte sollten für jede der vier beteiligten Zielklassen getrennt ihr emotionales Erleben beim Unterrichten beurteilen (z. B. «In dieser Klasse macht mir Unterrichten Freude»). Die Quantifizierung der Emotionsitems erfolgte über vierstufige Likert-Zustimmungsskalen (mit den Alternativen «trifft nicht zu», «trifft eher nicht zu», «trifft eher zu», «trifft zu»). Der dritte Teil der Vorbefragung umfasste Angaben zur Lehrkraft selbst (Alter, Geschlecht, Dienstjahre) sowie zu den vier beteiligten Klassen (Stufe, Größe, Anzahl Mädchen/Jungen sowie subjektiv eingeschätztes Leistungsniveau, klassifiziert in 1, «eher niedrig», 2, «mittel», 3, «hoch»).

In den Tagebuch-Erhebungen beurteilten die Lehrkräfte anhand folgender Einzelitems ihr emotionales Erleben in den jeweiligen Unterrichtsstunden: «Diese Stunde hat mir Spaß gemacht», «Ich war in dieser Stunde nervös und angespannt» und «Ich habe mich in dieser Stunde geärgert». Außerdem bewerteten sie ihren Eindruck vom Unterrichtsgeschehen entlang folgender drei Items: «Die Schüler waren in der heutigen Stunde motiviert», «Die Schüler waren heute diszipliniert» und «Die Schüler haben den Inhalt der heutigen Stunde verstanden». Eine Quantifizierung erfolgte auch hier über vierstufige Likert-Zustimmungsskalen.

Die am Gymnasium üblichen Stundentafeln sehen vor, dass Mathematik je nach Klassenstufe und Zweig zwischen drei und fünf Stunden pro Woche unterrichtet wird, Physik zwischen zwei und drei Stunden. In dieser Studie wurden im Durchschnitt $N=6.6$ Mathematikstunden $($ Min $=2$, Max $=10$ ) und $N=3.6$ Physik- bzw. NuT-Stunden (Min = 1 , Max =6) im Verlauf der zwei Schulwochen von den Lehrkräften beurteilt.

\subsection{Datenstruktur und Auswertungsstrategie}

Zum einen lagen im Rahmen der Vorbefragung Lehrerdaten bezüglich ihrer fachspezifischen unterrichtsbezogenen Emotionen vor. Das Ausmaß an Fachspezifität emotionalen Erlebens konnte anhand dieser Daten korrelativ bestimmt werden, vergleichbar mit dem Vorgehen in den Studien von Marsh (1990) oder Götz et al. (in press) zu Selbstkonzepten und Emotionen von Schülerinnen und Schülern.

Zum anderen entsprachen die klassenspezifischen Daten der Vorbefragung sowie die Tagebuchdaten Mehrebenenstrukturen, die ähnlich wie bei Raudenbush et al. (1992) und Ross et al. (1996) anhand des hierarchisch-linearen Modells ausgewertet werden konnten (vgl. Abbildung 1 für eine grafische Veranschaulichung der hierarchischen Datenstruktur). Von besonderer Bedeutung ist hier die Möglichkeit im hierarchischen linearen Modell, die jeweiligen Varianzanteile zu bestimmen, die zwischen den Einheiten der verschiedenen Ebenen liegen (vgl. z. B. Raudenbush \& Bryk, 2002).

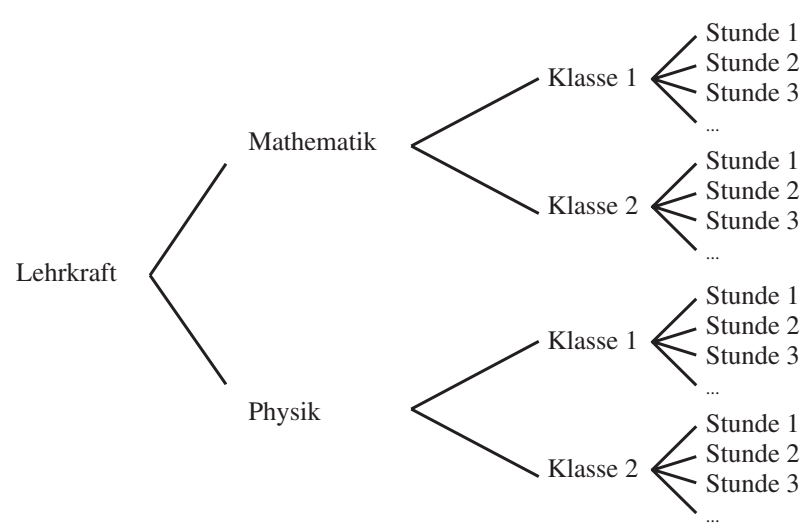

Abbildung 1. Grafische Veranschaulichung der hierarchischen Datenstruktur

Die Daten der Tagebuch-Erhebungen entsprachen einer Vier-Ebenen-Struktur: Die unterste Ebene (hier Ebene 0 genannt) umfasste die direkt nach jeder Unterrichtsstunde beurteilten emotionalen Befindlichkeiten aller beteiligten Lehrkräfte, was theoretisch insgesamt maximal 1200 Datenpunkten pro Emotion entsprach $(59$ Lehrkräfte $\times 2$ Fächer $\times 2$ Klassen $\times$ zwischen 1 und 10 Erhebungszeitpunkten pro Klasse). Aufgrund von unterschiedlichen Anzahlen fehlender Werte schwankte diese Anzahl leicht über die Emotionen hinweg; sie lag bei $N=1189$ für Freude, $N=$ 1192 für Angst und $N=1191$ für Ärger. Ebene 1 umfasste die jeweils über Klassen aggregierten Werte (59 Lehrkräfte $\times 2$ Fächer $\times 2$ Klassen, d. h. $N=236$ pro Emotion). Ebene 2 umfasste die fachweise aggregierten Werte (59 Lehrkräfte $\times 2$ Fächer, d. h. $N=118$ pro Emotion). Ebene 3 umfasste jeweils die Mittelwerte der drei Emotionen der Lehrkräfte $(N=59)$. 
Die klassenspezifischen Emotionsskalen der Vorbefragung lassen sich durch Aggregation in einer 3-Ebenen-Struktur darstellen: Auf unterster Ebene (Ebene 1) liegen auch hier die klassenspezifischen Werte $(N=236)$, auf Ebene 2 die jeweils fachweise aggregierten Werte $(N=118)$ auf Ebene 3 die Mittelwerte der Lehrkräfte $(N=59)$.

Die Mehrebenenanalysen wurden mit dem Programm MLwiN durchgeführt (Rasbash, Browne, Healy, Cameron \& Charlton, 2001). Diese Software ist im Moment die einzig kommerziell verfügbare, die das hierarchisch lineare Modell für mehr als drei Ebenen umsetzen kann. Die Varianzanalyse beim 4-Ebenen-Modell der Tagebuch-Daten im hierarchisch linearen Modell ist formal wie folgt darstellbar':

Das aktuelle emotionale Erleben in Stunde i in Klasse j im Fach k einer Lehrkraft 1 (z. B. Freude beim Unterrichten) bezeichnet mit Freude $\mathrm{ijkl}_{\mathrm{ijl}}$ - entspricht dem durchschnittlichen Freudeerleben dieser Lehrkraft in diesem Fach in dieser Klasse (Intercept $\mathrm{j}_{\mathrm{jk}}$ ) sowie der stundenspezifischen Abweichung $\mathrm{e}_{\mathrm{ijk} \mathrm{l}}$ davon: Freude $_{\mathrm{ijk} \mathrm{l}}=$ Intercept $_{\mathrm{jk} \mathrm{l}}+\mathrm{e}_{\mathrm{ijk} \mathrm{k}}$.

Ein positiver Wert von $\mathrm{e}_{\mathrm{ijkl}}$ würde bedeuten, dass die Lehrkraft in dieser Stunde in dieser Klasse mit mehr Freude unterrichtet hat als im Durchschnitt in diesem Fach in dieser Klasse. Dabei wird angenommen, dass die stundenspezifischen Abweichungen $\mathrm{e}_{\mathrm{ijk}}$ normalverteilt sind mit einem Mittelwert von 0 und einer Varianz $\Omega_{\mathrm{e}}$ : $\left[\mathrm{e}_{\mathrm{ijk}}\right] \sim \mathrm{N}\left(0, \Omega_{\mathrm{e}}\right]$.

Das durchschnittliche Freudeerleben einer Lehrkraft in einer Klasse wiederum lässt sich erklären durch das durchschnittliche Erleben dieser Lehrkraft beim Unterrichten des entsprechenden Faches sowie der klassenspezifischen Abweichung davon: Intercept $\mathrm{t}_{\mathrm{jk}}=$ Intercept $_{\mathrm{kl}}+\mathrm{u}_{\mathrm{jkl}}$.

Ein positiver Wert von $\mathrm{u}_{\mathrm{jkl}}$ würde beispielsweise bedeuten, dass die Lehrkraft in einer ihrer Physik-Klassen systematisch mehr Freude erlebt als in den anderen. Auch hier wird angenommen, dass diese klassenspezifischen Abweichungen $\mathrm{u}_{\mathrm{jkl}}$ normalverteilt sind mit einem Mittelwert von 0 und einer Varianz $\Omega_{\mathrm{u}}$. Sollte die Varianz $\Omega_{\mathrm{u}}$ signifikant von 0 abweichen, würde dies bedeuten, dass Lehrkräfte sich in Klassen gleichen Faches unterschiedlich fühlen, bedeutsame Anteile der intraindividuellen Variabilität emotionalen Erlebens also auf die spezifische Klasse zurückgehen.

Das durchschnittliche Erleben einer Lehrkraft in einem bestimmten Fach wiederum lässt sich erklären durch das durchschnittliche Erleben dieser Lehrkraft insgesamt sowie einer fachspezifischen Abweichung davon: Intercept $_{\mathrm{kl}}=$ Intercept $_{\mathrm{k}}+\mathrm{v}_{\mathrm{kl}}$.

Ein positiver Wert von $v_{\mathrm{kl}}$ würde dafür sprechen, dass eine Lehrkraft in einem ihrer beiden Fächer systematisch mehr Freude erlebt als im anderen. Auch hier gilt die Annahme $\left[\mathrm{v}_{\mathrm{kl}}\right] \sim \mathrm{N}\left(0, \Omega_{\mathrm{v}}\right]$. Die Größe der Varianz der fachspezifischen Abweichungen $\mathrm{O}_{\mathrm{v}}$ ist ein Indikator für das Ausmaß, zu dem sich Lehrkräfte in verschiedenen Unterrichtsfächern unterschiedlich fühlen (d. h. für die Anteile der intraindividuellen Variabilität, die auf das Fach zurückgehen).
Das durchschnittliche Freudeerleben einer Lehrkraft insgesamt lässt sich wiederum erklären durch Freude-Gesamtdurchschnitt aller Lehrkräfte sowie einer personspezifischen Abweichung davon: Intercept $t_{k}=$ Intercept $+f_{k}$.

Ein positiver Wert von $\mathrm{f}_{\mathrm{k}}$ würde in diesem Fall bedeuten, dass die jeweilige Lehrkraft systematisch mehr Freude beim Unterrichten erlebt als der Gesamtdurchschnitt aller teilnehmenden Lehrkräfte. Unter der Annahme von $\left[\mathrm{f}_{\mathrm{k}}\right] \sim \mathrm{N}\left(0, \Omega_{\mathrm{f}}\right]$ ist die Varianz $\Omega_{\mathrm{f}}$ also ein Indikator für die interindividuelle Varianz zwischen den Lehrkräften. Weicht sie signifikant von $0 \mathrm{ab}$, spricht dies dafür, dass das emotionale Erleben insgesamt von Lehrkraft zu Lehrkraft unterschiedlich ist.

Die Gesamtvarianz aller auf der untersten Ebene erfassten Daten pro Emotion (für die Tagebuchdaten: Die an die 1200 beurteilten Unterrichtsstunden aller Lehrkräfte; im 3Ebenen-Modell der Vorbefragungsdaten: Die 236 klassenspezifischen Summenscores zum emotionalen Erleben) setzt sich additiv aus den oben beschriebenen Varianzen auf den verschiedenen Ebenen zusammen $\left(\Omega_{\text {gesamt }}=\Omega_{\mathrm{e}}+\Omega_{\mathrm{u}}+\right.$ $\Omega_{\mathrm{v}}+\Omega_{\mathrm{f}}$ im Vierebenenmodell; $\Omega_{\text {gesamt }}=\Omega_{\mathrm{e}}+\Omega_{\mathrm{u}}+\Omega_{\mathrm{v}}$ im Dreiebenenmodell). Somit lassen sich nicht nur die absoluten Größen der jeweiligen Varianzen, sondern auch die jeweils auf den Ebenen anzusiedelnden Anteile zur Gesamtvarianz bestimmen.

Zusätzlich zur Bestimmung der Varianzanteile ist es im hierarchisch analytischen Modell möglich, Prädiktoren auf den verschiedenen Ebenen einzuführen. Im zweiten Schritt unserer Analysen untersuchten wir anhand solcher Mehrebenen-Regressionsanalysen den Einfluss der verschiedenen Person-, Klassen- und Situationsmerkmale auf das emotionale Erleben der Lehrkräfte.

\section{Ergebnisse}

Im Folgenden werden zunächst deskriptive Befunde zu unserer multimethodalen Erfassung von Freude, Angst und Ärger beim Unterrichten dargestellt. Dann werden die Ergebnisse der Mehrebenen-Regressionsanalysen für die Bestimmung der Varianzanteile auf den verschiedenen Ebenen (Person, Fach, Klasse, Unterrichtsstunde) präsentiert und in einem dritten Schritt die Vorhersagemodelle für die drei Emotionen durch Person-, Klassen- und Stundenmerkmale dargelegt.

Tabelle 1 zeigt die internen Konsistenzen der in der Vorbefragung durch die Lehrkräfte insgesamt sechs mal beurteilten Multi-Itemskalen zu Freude, Angst und Ärger beim Unterrichten (zwei mal fachspezifisch, vier mal klassenspezifisch). Alle so erfassten Skalen zum emotionalen Erleben beim Unterrichten waren zufrieden stellend reliabel (alle Cronbach's $\alpha \geq$.70). Die Tabelle zeigt auch die Mittelwerte und Standardabweichungen dieser Skalen. Insgesamt stimm-

1 Analog das 3-Ebenen-Modell der $N=236$ klassenspezifischen Daten der Vorbefragung, wobei hier die unterste Ebene wegfällt. 
Tabelle 1

Interne Konsistenzen (Cronbach's $\alpha$ ), Mittelwerte und Standardabweichungen der Multi-Itemskalen zum fachspezifischen und klassenspezifischen Erleben von Freude, Angst und Ärger beim Unterrichten (Vorbefragung).

\begin{tabular}{lccccccccc}
\hline Erfassungsbezug & \multicolumn{3}{c}{ Freude (4 Items) } & \multicolumn{3}{c}{ Angst (3 Items) } & \multicolumn{3}{c}{ Ärger (3 Items) } \\
& $\alpha$ & $M W$ & $S D$ & $\alpha$ & $M W$ & $S D$ & $\alpha$ & $M W$ & $S D$ \\
\hline Mathematik & .81 & 3.38 & .50 & .71 & 1.35 & .42 & .73 & 1.56 & .49 \\
Physik & .90 & 3.27 & .62 & .83 & 1.61 & .58 & .72 & 1.50 & .49 \\
Klasse 1 (Mathematik) & .90 & 3.21 & .69 & .90 & 1.33 & .56 & .82 & 1.66 & .61 \\
Klasse 2 (Mathematik) & .87 & 3.24 & .66 & .84 & 1.32 & .50 & .89 & 1.54 & .69 \\
Klasse 3 (Physik/NuT) & .92 & 3.10 & .64 & .71 & 1.35 & .42 & .74 & 1.57 & .62 \\
Klasse 4 (Physik/NuT) & .93 & 3.21 & .73 & .88 & 1.39 & .60 & .87 & 1.53 & .72 \\
\hline
\end{tabular}

Anmerkung. NuT steht für das in der Unterstufe unterrichtete Fach «Natur und Technik».

ten die Lehrkräfte den Items, die das Erleben von Freude beim Unterrichten thematisierten, stärker zu als denen, die Angst und Ärger erfassten. Das gleiche Bild ergab sich bei den Tagebuch-Erhebungen: Über alle Stunden hinweg ergaben sich hier Mittelwerte von 3.07 für Freude $(S D=.84), 1.24$ für Angst $(S D=.56)$ und 1.46 für Ärger $(S D=.76)$. Insgesamt gaben die Lehrkräfte in den Tagebüchern in $78 \%$ aller Stunden an, dass ihnen die Stunde Spaß gemacht habe, in $12 \%$ der Stunden gaben sie an, sich geärgert zu haben und in nur $5 \%$ der Stunden berichteten sie nervös und angespannt gewesen zu sein (jeweils Häufigkeiten der Antwortalternative «trifft eher zu» oder «trifft zu» bei diesen Items). Letztere verteilten sich jedoch nicht gleichmäßig über alle Lehrkräfte, sondern konzentrierten sich auf einige wenige Einzelpersonen: $5 \%$ der befragten Lehrkräfte gaben in einem nicht unerheblichen Anteil ihrer Stunden an (mindestens $37 \%$ ), angespannt und nervös gewesen zu sein.

Wir hatten angenommen, dass die Summe der über einen längeren Zeitraum hinweg gemittelten emotionalen States aus den Tagebüchern die im Fragebogen angegebenen Trait-Emotionen reflektieren. Tatsächlich ergaben sich enge Zusammenhänge zwischen dem im Fragebogen und im Tagebuch erfassten emotionalen Erleben: Die PearsonKorrelationen lagen bei $r=.79$ für Freude, $r=.48$ für Angst und $r=.68$ für Ärger.

Zur Überprüfung der Annahme, dass emotionales Erleben beim Unterrichten fachspezifisch ist, betrachteten wir zunächst die Korrelationen zwischen den in der Vorbefragung erfassten Skalen zum emotionalen Erleben in Mathematik und Physik. Entgegen unserer Hypothese, dass Lehrkräfte ihr Erleben von Freude, Angst und Ärger in Mathematik und Physik unterschiedlich bewerten, waren diese Korrelationen recht hoch: Sie lagen für Freude bei $r=.70$ und für Angst bei $r=.74$. Als etwas schwächer erwies sich der Zusammenhang bei Ärger $(r=.57)$.

Darüber hinaus verfolgten wir den oben beschriebenen hierarchisch linearen Ansatz, um die Anteile der Variabilität emotionalen Erlebens zu bestimmen, die auf Person, Fach und Klasse zurückgehen. Zunächst betrachteten wir für jede Emotion separat auf unterster Ebene die in der Vorbefragung erfassten 236 klassenspezifischen Summenscores zum emotionalen Erleben beim Unterrichten (Level
1: Klassen), auf der zweiten Ebene die jeweils innerhalb der Lehrkräfte gemittelten Werte pro Fach (Level 2: Fächer, $N=118$ ) und auf oberster Ebene die über beide Fächer gemittelten Werte der Lehrkräfte (Level 3: Lehrkräfte, $N=59$ ). Tabelle 2 (linke Seite, Vorbefragung) zeigt die durch MLwiN ermittelten Varianzen, die auf den jeweiligen Ebenen liegen. Die Signifikanz der jeweiligen Varianzen lässt sich anhand der in Tabelle 2 ebenfalls gezeigten Standardfehler beurteilen: Ist der geschätzte Koeffizient mindestens 1.96/2.58 mal so groß wie sein Standardfehler, weicht die Varianz signifikant $(p<.05 / .01)$ von Null ab (Wald Test). Bei Freude zeigte sich, dass die Gesamtvarianz zu jeweils etwa gleichen Teilen auf Unterschiede zwischen den Lehrkräften und auf Unterschiede innerhalb der Lehrkräfte zwischen ihren jeweiligen Klassen zurückging. Die Varianz in den berichteten Werten der Angst ging dagegen zu einem größeren Anteil (57\%) auf Unterschiede zwischen den Lehrkräften zurück und auf einen geringeren Anteil (43\%) auf die intraindividuelle Variation innerhalb der Lehrkräfte in den verschiedenen Klassen; bei Ärger war es umgekehrt. Bei keiner der drei Emotionen lagen bedeutsame Varianzanteile zwischen den beiden Fächern.

Auf analoge Weise analysierten wir die Daten des im Tagebuch geäußerten emotionalen Erlebens beim Unterrichten (vgl. Tabelle 2, rechte Seite). Hier lagen auf unterster Ebene (in Tabelle 2 Level 0 genannt) die verschiedenen durch die Lehrkräfte beurteilten Unterrichtsstunden $(N=$ 1189 für Freude, $N=1192$ für Angst und $N=1191$ für Ärger), auf der nächsten Ebene die jeweils klassenweise aggregierten Werte (Level 1, $N=236$ ), auf der nächsten Ebene die innerhalb der Lehrkräfte fachweise aggregierten Werte (Level 2, $N=118$ ), und auf der höchsten Ebene jeweils die Mittelwerte der Lehrkräfte (Level 3, $N=59$ ). Auch diese Analysen wurden jeweils für jede Emotion separat durchgeführt. Wie bei der State-Erhebung zu erwarten war, gab es bei allen Emotionen eine recht starke Variabilität über die Erhebungs-Zeitpunkte hinweg. Darüber hinaus variierten die durchschnittlich berichteten Emotionen innerhalb der Lehrkräfte systematisch über die verschiedenen Klassen hinweg. Auch bei dieser Analyse lag jedoch keine systematische Varianz zwischen dem mittleren emotionalen Erleben in den beiden Fächern. Die Lehr- 
Tabelle 2

Varianzanteile, die auf Person, Fach, Klasse und Unterrichtsstunde zurückgehen

\begin{tabular}{|c|c|c|c|c|c|c|}
\hline & \multicolumn{6}{|c|}{ Erhebungsmethode } \\
\hline & \multicolumn{3}{|c|}{ Vorbefragung } & \multicolumn{3}{|c|}{ Tagebuch } \\
\hline & $\begin{array}{l}\text { Absolute } \\
\text { Varianz }\end{array}$ & $S E$ & $\begin{array}{l}\text { Varianz- } \\
\text { anteil (\%) }\end{array}$ & $\begin{array}{l}\text { Absolute } \\
\text { Varianz }\end{array}$ & $S E$ & $\begin{array}{l}\text { Varianz- } \\
\text { anteil }(\%)\end{array}$ \\
\hline \multicolumn{7}{|l|}{ Freude } \\
\hline Level 3: Person, $\Omega\left(f_{1}\right)$ & .22 & .05 & 48 & .22 & .05 & 32 \\
\hline Level 2: Fach, $\Omega\left(\mathrm{v}_{\mathrm{kl}}\right)$ & .00 & .00 & 0 & .00 & .00 & 0 \\
\hline Level 1: Klasse, $\Omega\left(\mathrm{u}_{\mathrm{jk} 1}\right)$ & .24 & .03 & 52 & .07 & .02 & 11 \\
\hline Level 0: Unterrichtsstunde, $\Omega\left(\mathrm{e}_{\mathrm{ijkl}}\right)$ & & & & .40 & .02 & 57 \\
\hline \multicolumn{7}{|l|}{ Angst } \\
\hline Level 3: Person, $\Omega\left(f_{1}\right)$ & .16 & .03 & 57 & .08 & .02 & 25 \\
\hline Level 2: Fach, $\Omega\left(\mathrm{v}_{\mathrm{kl}}\right)$ & .00 & .00 & 0 & .00 & .00 & 0 \\
\hline Level 1: Klasse, $\Omega\left(\mathrm{u}_{\mathrm{jkl}}\right)$ & .12 & .01 & 43 & .02 & .01 & 6 \\
\hline Level 0: Unterrichtsstunde, $\Omega\left(\mathrm{e}_{\mathrm{ijkl}}\right)$ & & & & .20 & .01 & 69 \\
\hline \multicolumn{7}{|l|}{ Ärger } \\
\hline Level 3: Person, $\Omega\left(\mathrm{f}_{\mathrm{l}}\right)$ & .17 & .04 & 38 & .09 & .02 & 16 \\
\hline Level 2: Fach, $\Omega\left(\mathrm{v}_{\mathrm{kl}}\right)$ & .00 & .00 & 0 & .00 & .00 & 0 \\
\hline Level 1: Klasse, $\Omega\left(\mathrm{u}_{\mathrm{jk}}\right)$ & .26 & .03 & 62 & .08 & .02 & 14 \\
\hline Level 0: Unterrichtsstunde, $\Omega\left(\mathrm{e}_{\mathrm{ijkl}}\right)$ & & & & .41 & .02 & 71 \\
\hline
\end{tabular}

kräfte wiederum unterschieden sich systematisch voneinander in ihrem Erleben von Freude, Angst und Ärger.

Um diese Analysen mit denen der Vorbefragung vergleichen zu können, betrachteten wir zusätzlich den (prozentualen) Anteil der Varianz, der auf Person und Klasse zurückgeht, wenn man die stundenspezifische Variabilität ausklammert. Die Verhältnisse der prozentualen Varianzanteile Person zu Klasse entsprachen bei Freude 75:25, bei Angst 80:20 und bei Ärger 53:47.

In einem zweiten Analyseschritt führten wir auf den entsprechenden Ebenen Prädiktoren des Erlebens von Freude, Angst und Ärger ein. Da sich das Unterrichtsfach nicht als signifikante Varianzquelle erwiesen hatte, wurde hierzu lediglich eine 3-Ebenen-Struktur modelliert (Stunden geschachtelt in Klassen geschachtelt in Lehrkräften). Die Ergebnisse dieser Regressionsanalysen sind in Tabelle 3 dargestellt. In diesen Analysen kontrollierten wir das Geschlecht der Lehrkraft sowie die Klassenstufen der beteiligten Klassen (in Form von Dummyvariablen mit Mittelstufe [Klassenstufen 7 mit 10] als Referenzkategorie); diese wirkten sich jedoch erwartungsgemäß nicht auf das emotionale Erleben aus.

Entgegen unserer Erwartung gab es keine signifikanten Zusammenhänge zwischen Lehr-Erfahrung (Zahl der Dienstjahre) und den Emotionen. Hypothesenkonform zeigten sich jedoch signifikante Zusammenhänge zwischen den Kontrollüberzeugungen der Lehrkräfte und ihrem emotionalen Erleben: Je stärker die Lehrkräfte überzeugt waren, auf die Erreichung von Unterrichtszielen Einfluss nehmen zu können, desto mehr Freude, weniger Angst und weniger Ärger erlebten sie insgesamt über alle Klassen und Stunden hinweg.

Auf Klassenebene (d. h. innerhalb von Lehrkräften) hing das wahrgenommene Leistungsniveau der Klassen positiv mit dem Erleben von Freude, negativ mit dem Erleben von Ärger, aber nicht mit dem Erleben von Angst zusammen. Auch die Größe der Klassen erwies sich systematisch als prädiktiv für das emotionale Erleben: Größere Klassen führen zu verringerter Freude und erhöhtem Ärger beim Unterrichten, Angst blieb von der Klassengröße unbeeinflusst. Der Anteil an Jungen in der Klasse hing leicht positiv mit dem Erleben von Ärger zusammen.

Auf Stundenebene stand das durch die Lehrkräfte wahrgenommene Verständnis der Schüler in positivem Zusammenhang mit der Freude und in negativem Zusammenhang mit dem Ärger beim Unterrichten. Auch der Eindruck von Motiviertheit auf Seiten der Schüler bewirkte erhöhte Freude und geringeren Ärger bei den Lehrkräften, während das Erleben von Angst auch hiervon unbeeinflusst blieb. Die Wahrnehmung der Lehrkraft, die Schüler seien in den jeweiligen Stunden diszipliniert gewesen, stand in einem engem positiven Zusammenhang mit dem Erleben von Freude beim Unterrichten, deutliche negative Zusammenhänge fanden sich sowohl mit der Angst als auch dem Ärger.

\section{Diskussion}

Ziel dieser Studie war es, das Ausmaß an Variabilität des Erlebens von Freude, Angst und Ärger beim Unterrichten zu ermitteln, das auf die Lehrerpersönlichkeit, das Unterrichtsfach und die jeweils unterrichtete Klasse zurückgeht. Dazu erfassten wir Freude, Angst und Ärger zum einen anhand von Multi-Itemskalen in verschiedenen Spezifizie- 
Tabelle 3

Multiple Mehrebenen-Regressionen zur Vorhersage des Erlebens von Freude, Angst und Ärger beim Unterrichten

\begin{tabular}{lccc}
\hline & Freude & Angst & Ärger \\
\hline Personebene & & & \\
Geschlecht & -.11 & .13 & .10 \\
Lehr-Erfahrung & .01 & .02 & -.04 \\
Kontrollüberzeugung & $.30 * *$ & $-.22 * *$ & $-.19 * *$ \\
Klassenebene & & & \\
Wahrg. Leistungsniveau & $.15 * *$ & .04 & $-.11^{*}$ \\
Größe der Klasse & $-.11 *$ & .09 & $.21 * *$ \\
\% Jungen in Klasse & -.05 & .02 & $.13 *$ \\
Unterstufe (Dummy) & .03 & -.02 & .00 \\
Oberstufe (Dummy) & -.01 & .03 & .01 \\
Stundenebene & & & \\
Wahrg. Verständnis & $.18 * *$ & -.06 & $-.12^{* *}$ \\
Wahrg. Motivation & $.27 * *$ & -.07 & $-.12^{* *}$ \\
Wahrg. Disziplin & $.28 * *$ & $-.23 * *$ & $-.42^{* *}$ \\
\hline
\end{tabular}

$* p<.05 ; * * p<.01$

Anmerkungen. Die abhängigen Variablen wurden auf der untersten Ebene $z$-standardisiert; die unabhängigen Variablen wurden auf ihren jeweiligen Ebenen $z$-standardisiert. Koeffizienten sind also innerhalb von Ebenen (hier auch über die Emotionen hinweg), aber nicht über Ebenen hinweg, vergleichbar. Geschlecht wurde negativ für Männer, positiv für Frauen kodiert, d. h. positive Koeffizienten bedeuten höhere Werte für Frauen. «Unterstufe» repräsentiert die Klassenstufen 5 und 6, «Oberstufe» die Klassenstufen 11, 12 und 13. Mittelstufe (Klassen 7 mit 10) repräsentiert die Referenzkategorie. Die Prädiktoren wurden jeweils gruppenmittelzentriert, da die reinen Effekte innerhalb der Ebenen von Interesse waren. Diese Analysen basieren nur auf $N=55$ Lehrkräften, $N=211$ Klassen und $N=1.082$ Stunden aufgrund einzelner fehlender Angaben.

rungsgraden: Hinsichtlich des Unterrichtens von Mathematik und Physik und hinsichtlich der verschiedenen von den Lehrkräften unterrichteten Klassen. Zum anderen bearbeiteten die Lehrkräfte über zwei Wochen hinweg ein Tagebuch, in dem sie direkt im Anschluss an die Unterrichtsstunden ihre Freude, Angst und ihren Ärger in der jeweiligen Stunde beurteilten. Wir gingen davon aus, mit diesen beiden Methoden grundsätzlich insofern Gleiches zu erfassen, als sich die im Fragebogen erfassten emotionalen Traits auch in den innerhalb von Personen über diverse Situationen hinweg gemittelten emotionalen States widerspiegeln sollten. Mittelhohe Korrelationen über die Methoden hinweg bestätigen diese Annahme. Auch lieferten die auf den beiden verschiedenen Methoden basierenden Analysen der inter- und intraindividuellen Varianzanteile des emotionalen Erlebens ähnliche Ergebnisse. Wir schließen daraus, mit beiden Methoden gleichermaßen einen geeigneten Zugang zur Erfassung der Konstrukte Unterrichtsfreude, -angst und -ärger von Lehrkräften gefunden zu haben.

In einem weiteren Analyseschritt zielten wir außerdem darauf ab, den Einfluss von Person-, Klassen- und Stundenmerkmalen auf das emotionale Erleben beim Unterrichten zu untersuchen. Dazu erfassten wir die allgemeinen Kontrollüberzeugungen der Lehrkräfte hinsichtlich der Einflussmöglichkeiten auf das Erreichen von Unterrichtszielen, Eigenschaften der Klassen (Größe, Anteil an Jungen, Leistungsniveau) sowie als weiteren Teil der Tagebuchbefragung die Eindrücke der Lehrkraft hinsichtlich des Unterrichtsverlaufs (Schülerverständnis, -motivation und -disziplin in den jeweiligen Stunden).

\subsection{Person-, Fach- und Klassenspezifität unterrichtsbezogener Emotionen von Lehrkräften}

Unsere mehrebenenanalytische Auswertung der inter- und intraindividuellen Variabilität emotionalen Erlebens legt nahe, dass neben Unterschieden im Erleben von Freude, Angst und Ärger zwischen Lehrkräften auch bedeutsame Variabilität innerhalb der Lehrkräfte vorhanden ist, welche je nach Emotion unterschiedlich ausfällt. Unsere Hypothese, dass das Erleben von Freude, Angst und Ärger beim Unterrichten personspezifisch ist, kann daher grundsätzlich angenommen werden, jedoch ist das Ausmaß an Personspezifität für die verschiedenen Emotionen unterschiedlich stark ausgeprägt. Die Tagebuchbefragungen legten nahe, dass der Anteil der Gesamtvarianz aller direkt nach Unterrichtsstunden beurteilten Emotionen (ohne die state-spezifische Varianz), der auf personspezifische Unterschiede zurückging, für Freude bei $75 \%$, für Angst bei $80 \%$, für Ärger aber nur bei $53 \%$ lag.

Anhand der Betrachtung des klassenspezifischen Erlebens von Freude, Angst und Ärger beim Unterrichten, das jede Lehrkraft im Fragebogen für vier ihrer Klassen beurteilt hat, ermittelten wir für die Freude einen Anteil von $48 \%$, der zwischen Lehrkräften lag, für Angst einen Anteil von $57 \%$ und für den Ärger einen Anteil von nur $38 \%$. Dass das Verhältnis von intraindividueller zu interindividueller Varianz bei dieser Analyse bei allen Emotionen geringer war als bei der Analyse der Tagebuchdaten führen wir darauf zurück, dass die wiederholte klassenspezifische Erfassung im Fragebogen («Wie fühlen Sie sich in Klasse 1/2/3/4?») die Unterschiedlichkeit zwischen den Klassen für die Probanden besonders hervorgehoben hat, was zu Kontrasteffekten geführt haben könnte. Insgesamt aber ergibt sich aus beiden Analysestrategien ein eindeutiges Bild hinsichtlich der Personspezifität emotionalen Erlebens beim Unterrichten: Angst ist am stärksten personspezifisch, gefolgt von Freude. Das Erleben von Ärger beim Unterrichten dagegen scheint mindestens ebenso personspezifisch (im Sinne einer habituellen Neigung, Ärger zu erleben) wie kontextspezifisch zu sein.

Darüber hinaus hatten wir angenommen, dass sowohl das Unterrichtsfach (Mathematik vs. Physik) als auch die jeweilig unterrichtete Klasse eine Rolle spielen. Tatsächlich sprechen unsere Daten dafür, dass bedeutsame Anteile der intraindividuellen Variation des emotionalen Erlebens auf die Verschiedenartigkeit der Klassen zurück zu 
gehen scheint. Diese liegen in einer vergleichbaren Größenordnung wie die in den methodisch ähnlich aufgebauten Studien zu Lehrer-Selbstwirksamkeit von Raudenbush et al. (1992) sowie Ross et al. (1996).

Unsere Daten sprechen also dafür, dass bedeutsame Anteile der intraindividuellen Variabilität des emotionalen Erlebens von Lehrkräften zwischen Klassen liegen - eine Fachspezifität lässt sich (bei Kontrolle der Klassenspezifität) anhand unserer Daten jedoch nicht nachweisen. Zwischen den Fächern Mathematik und Physik lag weder bei der Analyse der Daten der Vorbefragung, noch der der Tagebücher systematische intraindividuelle Varianz. Das heißt, welche Klasse Lehrkräfte unterrichten, wirkt sich in nicht unerheblichem Maße auf ihr Erleben von Freude, Angst und insbesondere Ärger aus, beim Unterrichten von Mathematik und Physik scheinen sie sich aber insgesamt ähnlich zu fühlen. Dafür sprechen auch die recht hohen Korrelationen zwischen dem in der Vorbefragung getrennt für die beiden Fächer beurteilten Erleben von Freude, Angst und Ärger. Diese lagen um .70 (mit der Ausnahme von Ärger, der auch in dieser Hinsicht kontextspezifischer ausgeprägt war, mit Korrelationen um .60) und damit deutlich höher, als im Bereich Schüleremotionen und -selbstkonzepten berichtet wurde (mit Korrelationen maximal um .50; z. B. Götz et al., in press; Marsh, 1990). Wir hatten eine Fachspezifität emotionalen Erlebens von Lehrkräften unter anderen deswegen erwartet, weil Riggs und Enochs (1990) eine fachspezifische Erfassung von LehrerSelbstwirksamkeit in den Naturwissenschaften vs. in anderen Fächern angeregt hatten. Tatsächlich aber gibt es unseres Wissens bisher keine Studien zu intraindividueller Variabilität von Lehrer-Selbstwirksamkeit aufgrund von unterschiedlichen unterrichteten Fächern. Ein Grund hierfür könnte darin liegen, dass in vielen Ländern, u. a. in den USA, Lehrkräfte jenseits des Primarbereichs selten unterschiedliche Fächer unterrichten und meistens auch gar nicht in mehreren Fächern ausgebildet sind. Tatsächlich scheinen sich die Fächer Mathematik und Physik zumindest in instruktionspsychologischer Hinsicht so zu gleichen, dass Lehrkräfte das Unterrichten in diesen beiden Fächern sehr ähnlich erleben. Gegenstand zukünftiger Studien sollte jedoch die Frage sein, ob das auch für das Unterrichten in weniger eng verwandten Fächern gilt - insbesondere beispielsweise im Primarbereich, wo eine Lehrkraft in der Regel die gesamte Bandbreite möglicher Fächer unterrichtet, oder im Hauptschulbereich, wo Lehrkräfte alle Fächer unterrichten müssen, auch solche, in denen sie nicht vertieft ausgebildet sind.

\subsection{Prädiktoren des Erlebens von Freude, Angst und Ärger beim Unterrichten}

In Anknüpfung an unsere Befunde, dass Angst in stärkerem Maße als Freude und Ärger personspezifisch ist, fällt auf, dass die auf Lehrerebene angesiedelte Kontrollüberzeugung ein bedeutsamer Prädiktor für Angst ist, während Klassenmerkmale und auch Eindrücke der Lehrkräfte hinsichtlich der konkreten Unterrichtsstunden für das Erleben von Angst keine oder eine nur geringe Rolle zu spielen scheinen. Je stärker eine Lehrkraft davon überzeugt ist, dass das Erreichen von Unterrichtszielen durch externe Gegebenheiten vorgegeben ist und nicht in ihrer Macht liegt, desto mehr Angst erlebt sie beim Unterrichten - ein Befund, der mit appraisaltheoretischen Annahmen zur Entstehung von Angst im Einklang steht (z. B. Pekrun, 2006; Roseman et al., 1996). Hat eine Lehrkraft zudem noch den Eindruck, dass Schülerinnen und Schüler in konkreten Unterrichtsstunden wenig diszipliniert sind, erhöht das ihre Anspannung und Nervosität. Der von Hart (1987) bei Lehramtskandidaten beschriebene Zusammenhang zwischen Disziplinschwierigkeiten und Angst lässt sich also auch in unserer Lehrerstichprobe replizieren, die eine breite Altersspanne umfasste.

Für Freude und Ärger spielen Prädiktoren auf Person-, Klassen- und Stundenebene eine Rolle. Qualitative Befunde zu Ursachen von Lehrerärger (z. B. Hargreaves, 2000) bestätigend, erwiesen sich die wahrgenommenen Disziplinschwierigkeiten während des Unterrichts auch in unserer Studie als wichtigster Faktor für das Erleben von Ärger. Zusätzlich scheint aber auch der Eindruck der Lehrkraft, die Schüler seien während der Stunden motiviert und sie würden den Unterrichtsstoff verstehen, den Ärger während der Stunden zu senken. Für die Entstehung von Freude beim Unterrichten scheinen dagegen wahrgenommene Disziplin und wahrgenommene Motivation der Schülerinnen und Schüler in den Unterrichtsstunden gleichermaßen beizutragen, aber auch das wahrgenommene Verständnis der Schüler hat einen zusätzlichen positiven Effekt auf die Unterrichtsfreude der Lehrkraft.

In Übereinstimmung mit unseren Annahmen berichten Lehrkräfte jenseits ihrer Wahrnehmung hinsichtlich Verständnis, Motivation und Disziplin in den konkreten Unterrichtsstunden in größeren und leistungsschwächeren Klassen weniger Freude und mehr Ärger. Der Anteil an Jungen in Klassen wirkt sich zudem verstärkend auf den Ärger der Lehrkräfte aus. Es ist bemerkenswert, dass diese Klassenmerkmale unter Kontrolle der konkreten Eindrücke vom Unterrichtsverlauf einen bedeutsamen Einfluss haben. Es wäre denkbar gewesen, dass sich die Größe der Klasse auf Verständnis-, Motivations- und Disziplinniveau beim Unterrichten auswirken und die Emotionen der Lehrkräfte somit nur indirekt beeinflussen würden, da in Studien wiederholt gezeigt werden konnte, dass Schüler in kleineren Klassen engagierter, aufmerksamer und disziplinierter sind als in großen Klassen (z. B. Finn \& Achilles, 1999; Smith \& Glass, 1979). Große Klassen scheinen also noch über diese Faktoren hinaus für Lehrkräfte emotional belastende Charakteristiken aufzuweisen.

Im Einklang mit appraisaltheoretischen Annahmen scheint zudem die Kontrollüberzeugung von Lehrkräften für interindividuelle Unterschiede im Erleben von Freude und Ärger beim Unterrichten verantwortlich zu sein: Lehrkräfte mit internalen Kontrollüberzeugungen berichten in unserer Studie größere Unterrichtsfreude und geringeren Ärger beim Unterrichten. 


\subsection{Bedeutung für die Unterrichtspraxis}

Insgesamt sprechen unsere Daten dafür, dass emotionales Erleben bei der Mehrzahl der Lehrkräfte vorwiegend positiv geprägt ist. Es kam jedoch bei Einzelpersonen (insgesamt ca. $5 \%$ der Teilnehmerinnen und Teilnehmer) durchaus vor, dass ein erheblicher Anteil ihrer Unterrichtsstunden durch Nervosität und Angst geprägt war. Während also insbesondere die Emotion Angst im Unterrichtsalltag vieler Lehrkräfte relativ schwach ausgeprägt zu sein scheint, gibt es doch vereinzelt Lehrkräfte, die durchaus mit Angstgefühlen während des Unterrichtens zu kämpfen haben. «Ä rger» ist dagegen über alle Lehrkräfte hinweg in insgesamt mehr als $10 \%$ ihrer Stunden eine treffende Beschreibung ihres emotionalen Erlebens.

Die Erkenntnisse dieser Studie zur relativen Kontextspezifität und zu den Prädiktoren von Emotionen beim Unterrichten halten wir auch deswegen für bedeutsam, weil sich daraus konkrete Schlüsse für mögliche Interventionen ableiten lassen. Ist das emotionale Erleben vor allem klassenspezifisch, sollten Lehrkräfte angeleitet werden, die Eigenschaften bestimmter Klassen auszumachen, die diese Emotionen bei ihnen auslösen, und dementsprechend problemspezifische, klassenspezifische Maßnahmen ergreifen, um die Situation zu verbessern und damit ihr emotionales Erleben zu optimieren - dies gilt entsprechend unserer Daten insbesondere für Ärger. Unsere Analysen legen nahe, dass Disziplinschwierigkeiten in der Klasse eine wichtige Ursache von Lehrer-Ärger sind. Mit dem Ziel der Senkung von Ärger beim Unterrichten sollten durch die Lehrkräfte dementsprechend gezielt problematische Klassen identifiziert werden und dann klassenspezifische Interventionen, beispielsweise zur Verbesserung der Disziplin, angestrebt werden. Ist das emotionale Erleben dagegen in erster Linie eine Persönlichkeitseigenschaft, die nur wenig über die verschiedenen Klassen hinweg variiert, scheinen Interventionen auf Personebene funktional zu sein. In unserer Studie haben sich Kontrollüberzeugungen der Lehrkräfte hinsichtlich ihrer Einflussmöglichkeiten auf das Erreichen von Unterrichtszielen als bedeutsam für das personspezifische Erleben vor allem von Freude und Angst, aber auch von Ärger, erwiesen. Hier könnten dementsprechend beispielsweise Interventionen auf Personebene ansetzen.

Unsere Befunde sind schließlich nicht nur hinsichtlich des Wohlbefindens von Lehrkräften bedeutsam, sondern auch, weil wir davon ausgehen, dass sich das emotionale Erleben einer Lehrkraft auf ihr Unterrichtsverhalten auswirkt und somit den Lernerfolg von Schülerinnen und Schülern mitbestimmt (Frenzel et al., im Druck; Meyer \& Turner, 2002; Sutton \& Wheatley, 2003). Die vorliegende Studie gibt erste Hinweise zu Erfassungsmöglichkeiten und Antezedenzien von Lehreremotionen. Zukünftige Studien sollten untersuchen, inwieweit das emotionale Erleben von Lehrkräften mit der Qualität von Lehren und Lernen im Klassenzimmer im Zusammenhang steht.

\section{Literatur}

Armor, D., Conroy-Oseguera, P., Cox, M., King, N., McDonnell, L., Pascal, A., Pauly, E. \& Zellman, G. (1976). Analysis of the school preferred reading programs in selected Los Angeles minority schools. (Report No. R-2007-LAUSD). Santa Monica, CA: Rand Corporation (ERIC Document Reproduction Service No. 130 243).

Ashton, P. T. \& Webb, R. B. (1986). Making a difference: Teachers' sense of efficacy and student achievement. New York: Longman.

Bayerisches Staatsministerium für Unterricht und Kultus. (2005). Schule und Bildung in Bayern 2005: Statistische Übersichten. München: Bayerisches Staatsministerium für Unterricht und Kultus.

Brown, R. \& Gibson, S. (1982, April). Teachers' sense of efficacy: Changes due to experience. Paper presented at the annual meeting of the California Educational Research Association. Sacramento, CA.

Davies, J. \& Brember, I. (1995, September). Attitudes to school and the curriculum in year 2, year 4 and year 6: Changes over four years. Paper presented at the European Conference on Educational Research, Bath, UK.

Enochs, L. G., Smith, P. L. \& Huinker, D. (1999). Establishing factorial validity of the mathematics teaching efficacy beliefs instrument. School Science and Mathematics, 100, 194-202.

Finn, J. D. \& Achilles, C. M. (1999). Tennessee's class size study: Findings, implications, misconceptions. Educational Evaluation and Policy Analysis, 21, 97-109.

Frenzel, A. C., Götz, T. \& Pekrun, R. (im Druck). Ursachen und Wirkungen von Lehreremotionen. In M. Gläser-Zikuda \& J. Seifried (Hrsg.), Analyse und Förderung von Lehrerexpertise. Münster: Waxmann.

Götz, T., Frenzel, A.C., Pekrun, R. \& Hall, N.C. (2006). The domain specificity of academic emotional experiences, Journal of Experimental Education, 75, 5-29.

Götz, T., Frenzel, A. C., Pekrun, R., Hall, N. C. \& Lüdtke, O. (in press). Between- and within-domain relations of students' academic emotions. Journal of Educational Psychology.

Grimminger, M. (2005). Hey, Lehrer! Schulangst? Donauwörth: Auer.

Hargreaves, A. (2000). Mixed emotions: Teachers' perceptions of their interactions with students. Teaching and Teacher Education, 16, 811-826.

Hart, N. (1987). Student teachers' anxieties: Four measured factors and their relationships to pupil disruption in class. Education Research, 29, 12-18.

Hascher, T. (2004). Wohlbefinden in der Schule. Münster: Waxmann

Hedges, S. M., Jandorf, L. \& Stone, A. A. (1985). Meaning of daily mood assessments. Journal of Personality and Social Psychology, 48, 428-434.

Helmke, A. (2003). Unterrichtsqualität erfassen, bewerten, verbessern. Seelze: Kallmeyer.

Hoy, W. K. \& Woolfolk, A.E. (1993). Teachers' sense efficacy and the organizational health of schools. The Elementary School Journal, 93, 356-372.

Jones, S. M. \& Dindia, K. (2004). A meta-analytic perspective on sex equity in the classroom. Review of Educational Research, $74,443-471$. 
Kunter, M., \& Tsai, Y.-M., Brunner, M., \& Krauss, M. (2005, August). Enjoying teaching: Enthusiasm and teaching behaviors in secondary school mathematics teachers. Paper presented at the 11th Conference of the European Association for Research in Learning and Instruction (EARLI), Nicosia, Cyprus.

Lazarus, R. (1994). The stable and the unstable in emotion. In P. Ekman \& R. J. Davidson (Eds.), The nature of emotions: Fundamental questions (pp.79-85). Oxford: Oxford University Press.

Lazarus, R. S. Folkman, S. (1984). Stress, appraisal, and coping. New York: Springer.

Marsh, H. W. (1990). The structure of academic self-concept: The Marsh/Shavelson model. Journal of Educational Psychology, 82, 623-636.

Maslach, C. \& Jackson, S. E. (1986). Maslach Burnout Inventory. Palo Alto, CA: Consulting Psychologists Press.

Maslach C., Schaufeli W. B. \& Leiter M. P. (2001). Job burnout. Annual Review of Psychology, 52, 397-422.

Meyer, D. K. \& Turner, J.C. (2002). Discovering emotion in classroom motivation research. Educational Psychologist, 37, $107-114$.

Parkinson, B., Briner, R. B., Reynolds, S. Totterdell, P. (1995) Time frames for mood: Relations between momentary and generalized ratings of affect. Personality and Social Psychology Bulletin, 21, 881-339.

Payne, B. D. \& Manning, B. H. (1990). The effect of self-instructions on preservice teacher's anxiety about teaching. Contemporary Educational Psychology, 15, 261-267.

Pekrun, R. (2006). The control-value theory of achievement emotions: Assumptions, corollaries, and implications for educational research and practice. Educational Psychology Review, $18,315-341$

Pekrun, R., Frenzel, A.C., Götz, T. \& Perry, R.P. (2007). The control-value theory of achievement emotions: An integrative approach to emotions in education. In P. A. Schutz \& R. Pekrun (Eds.), Emotions in education (pp. 13-36). San Diego: Academic Press.

Pekrun, R. Götz, T., Titz, W. \& Perry, R.P. (2002). Academic emotions in students' self-regulated learning and achievement: A program of qualitative and quantitative research. Educational Psychologist, 37, 91-105.

Pekrun, R., Götz, T. \& Frenzel, A. C. (2005). Academic Emotions Questionnaire - Mathematics (AEQ-M) - User's manual. University of Munich: Department of Psychology.

Pekrun, R., Götz, T. \& Perry, R. P. (2005). Academic Emotions Questionnaire (AEQ) - User's manual. University of Munich: Department of Psychology.

Pigge, F. L. \& Marso, R. N. (1993, Februar). Outstanding teachers' sense of efficacy at four stages of career development. Paper presented at the Annual Conference of the Association of Teacher Educators, Los Angeles, CA.

Ramm, G., Prenzel, M., Baumert, J., Blum, W., Lehmann R., Leutner, D., Neubrand M., Pekrun, R., Rolff, H.-G., Rost, J. \& Schiefele U. (2006). PISA 2003. Dokumentation der Erhebungsinstrumente. Münster: Waxmann.

Rasbash, J., Browne, W., Healy, M. Cameron, B. \& Charlton, C. (2001). The MLwiN software Package version 1.10.0007 [Computer Software]. London: Institute of Education.

Raudenbush, S. W. \& Bryk, A. S. (2002). Hierarchical linear mo- dels. Applications and data analysis methods (2nd ed.). Thousand Oaks, CA: Sage.

Raudenbush, S. W., Rowan, B. \& Cheong, Y.F. (1992). Contextual effects on the self-perceived efficacy of High School teachers. Sociology of Education, 65, 150-167.

Riggs, I. M. \& Enochs, L. G. (1990). Toward the development of an elementary teacher's science teaching efficacy belief instrument. Science Education, 74, 625-637.

Roseman, I. J., Antoniou, A. A. \& Jose, P.E. (1996). Appraisal determinants of emotions: Constructing a more accurate and comprehensive theory. Cognition and Emotion, 10, 241-277.

Ross, J. A., Cousins, J. B. \& Gadalla, T. (1996). Within-teacher predictors of self-efficacy. Teaching and Teacher Education, $12,385-400$.

Schaarschmidt, U. (2004). Halbtagsjobber? Psychische Gesundheit im Lehrerberuf - Analyse eines veränderungsbedürftigen Zustandes. Weinheim: Beltz

Schmitz, B. (1987). Zeitreihenanalyse in der Psychologie: Verfahren zur Veränderungsmessung und Prozessdiagnostik. Weinheim: Beltz.

Shapson, S. M., Wright, E. N., Eason, G. \& Fitzgerald, J. (1980). An experimental study of the effects of class size. American Educational Research Journal, 17, 141-152.

Smith, M. L. \& Glass, G. V. (1979). Relationship of class-size to classroom processes, teacher satisfaction and pupil affect: A meta-analysis. San Francisco, CA: Far West Laboratories.

Steyer, R., Schmitt, R. \& Eid, M. (1999). Latent state-trait theory and research in personality and individual differences. European Journal of Personality, 13, 389-408.

Sutton, R.E. (2004). Emotional regulation goals and strategies of teachers. Social Psychology of Education, 7, 379-389.

Sutton, R.E. \& Wheatley, K.F. (2003). Teachers' emotions and teaching: A review of the literature and directions for future research. Educational Psychology Review, 15, 327-358.

Thomas, D. L. \& Diener, E. (1990). Memory Accuracy in the recall of emotions. Journal of Personality and Social Psychology, 59, 291-297.

Tschannen-Moran, M. \& Hoy, A. W. (2001). Teacher efficacy: Capturing an elusive construct. Teaching and Teacher Education, 17, 783-805.

Turner, J.C., Meyer, D. K., Midgley, C. \& Patrick, H. (2001, April). The relationship between teachers' affective discourse and students' approach and avoidance beliefs and behaviors in mathematics: What happens in high mastery/high performance classrooms? Paper presented at the annual meeting of the American Educational Research Association, Seattle, WA.

Zembylas, M. (2002). Constructing genealogies of teachers' emotions in Science teaching. Journal of Research in Science Teaching, 39, 79-103.

\section{Dr. Anne C. Frenzel}

Universität München

Department Psychologie

Leopoldstr. 13

D-80802 München

Tel. +49 89 2-180-6047

Fax +49 89 2-180-5250

E-mail frenzel@edupsy.uni-muenchen.de 